UCRL-ID-134061

\title{
3D Dislocation Dynamics: Stress-Strain Behavior and Hardening Mechanisms in FCC and $\mathrm{BCC}$ Metals
}

Hussein M. Zbib

Tomas Diaz de la Rubia

Moono Rhee

John P. Hirth

February 19, 1999

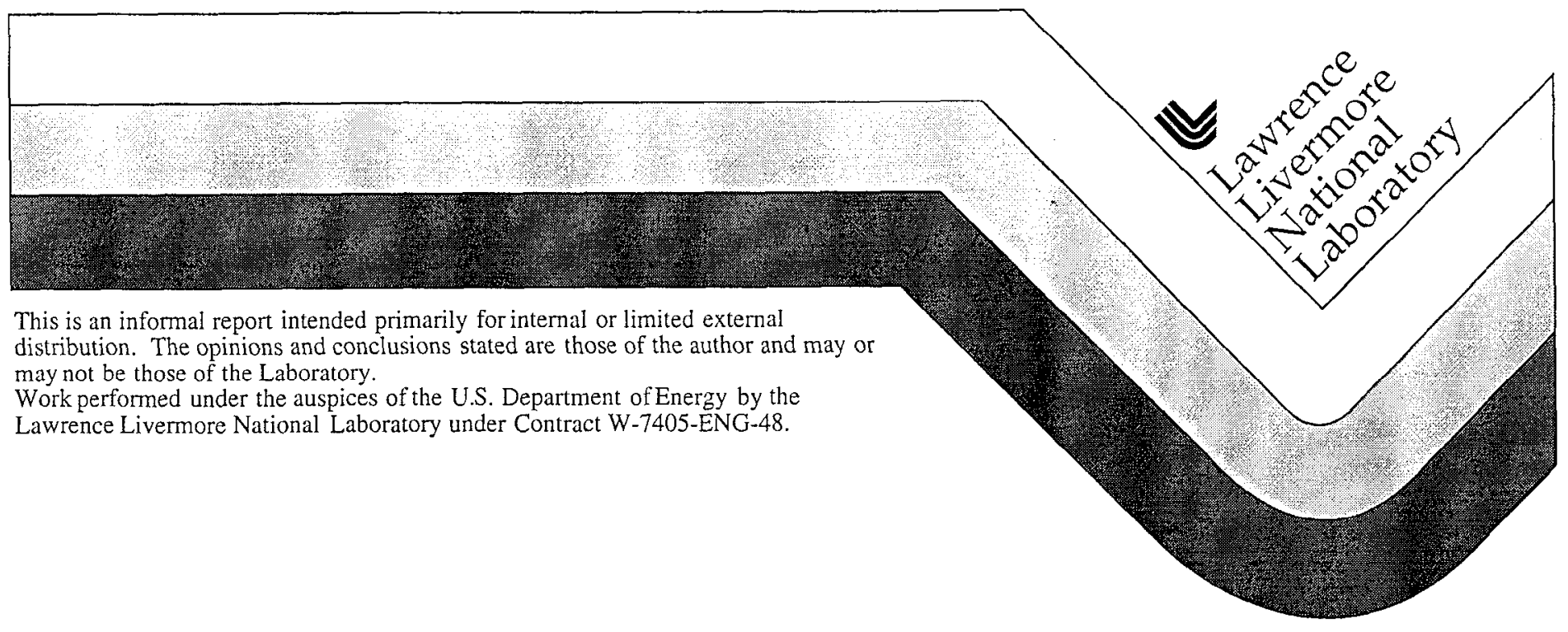




\section{DISCLAIMER}

This document was prepared as an account of work sponsored by an agency of the United States Government. Neither the United States Government nor the University of California nor any of their employees, makes any warranty, express or implied, or assumes any legal liability or responsibility for the accuracy, completeness, or usefulness of any information, apparatus, product, or process disclosed, or represents that its use would not infringe privately owned rights. Reference herein to any specific commercial product, process, or service by trade name, trademark, manufacturer, or otherwise, does not necessarily constitute or imply its endorsement, recommendation, or favoring by the United States Government or the University of California. The views and opinions of authors expressed herein do not necessarily state or reflect those of the United States Government or the University of California, and shall not be used for advertising or product endorsement purposes.

This report has been reproduced directly from the best available copy.

Available to DOE and DOE contractors from the Office of Scientific and Technical Information

P.O. Box 62, Oak Ridge, TN 37831

Prices available from (423) 576-8401

Availabie to the public from the National Technical Information Service

U.S. Department of Commerce

5285 Port Royal Rd.

Springfield, VA 22161 


\title{
3D Dislocation Dynamics:
}

\section{Stress-Strain behavior and Hardening Mechanisms in FCC and BCC Metals}

\author{
Hussein M. Zbib ${ }^{1}$, Tomas Diaz de La Rubia ${ }^{2}$, Moono Rhee ${ }^{2}$ and John P. Hirth ${ }^{1}$ \\ ${ }^{1}$ School of Mechanical and Materials Engineering \\ Washington State University, Pullman WA 99164-2920 \\ ${ }^{2}$ Lawrence Livermore National Laboratory \\ Livermore, CA 94550
}

\begin{abstract}
A dislocation dynamics (DD) model for plastic deformation, connecting the macroscopic mechanical properties to basic physical laws governing dislocation mobility and related interaction mechanisms, has been under development. In this model there is a set of critical reactions that determine the overall results of the simulations, such as the stress-strain curve. These reactions are, annihilation, formation of jogs, junctions, and dipoles, and cross-slip. In this paper we discuss these reactions and the manner in which they influence the simulated stressstrain behavior in fcc and bcc metals. In particular, we examine the formation (zipping) and strength of dipoles and junctions, and effect of jogs, using the dislocation dynamics model. We show that the strengths (unzipping) of these reactions for various configurations can be determined by direct evaluation of the elastic interactions. Next, we investigate the phenomenon of hardening in metals subjected to cascade damage dislocations. The microstructure investigated consists of small dislocation loops decorating the mobile dislocations. Preliminary results reveal that these loops act as hardening agents, trapping the dislocations and resulting in increased hardening.
\end{abstract}




\section{INTRODUCTION}

Constitutive modeling of deformation of metals under extreme loading conditions depends critically on our understanding of the relationship between the macroscopic mechanical properties and the underlying dislocation sub-structures. Such structures could be highly heterogeneous and include dislocation cells, slip bands, microshear bands, persistent slip bands and dislocation tangles, all of which are critical to material properties [1-7]. Understanding how these structures form and evolve and how they affect work hardening is, perhaps, one of the most difficult tasks which is still rife with controversy. The main difficulty has been in dealing with large numbers of dislocations. For example, Kuhlmann-Wilsdorf [7] has proposed that the structures can be understood as a progression of low energy thermodynamic states with something like a conventional phase transition taking place between carpet structures and 3D cell structures at the end of Stage II. However, Holt [8] proposed that dislocation structure evolution can be viewed in the spirit of spinodal decomposition by the introduction of local densities of diffusing dislocation populations. This approach has been adopted in later models. In particular, Walgraef and Aifantis [9] developed a model of dislocation patterning to describe the evolution of ordered structures in chemically reacting systems. In this model, several types of dislocation populations are introduced as density functions of position in space, with the evolution determined by diffusion and reaction terms. Although these reaction-diffusion schemes have been successful in modeling $2 \mathrm{D}$ dislocation patterns, they have a number of difficulties associated with determination of model parameters and they do not yet address realistic $3 \mathrm{D}$ dislocation configurations.

By viewing the dislocation structures problem as a dynamical system, one can establish a number of discrete models to understand the origin of dislocation structures in deformed crystals. Although the method was initiated over a decade ago, most of the original models were twodimensional and consisted of periodic cells each with dislocations of infinite length. These 2D models have provided some understanding of rules of interaction and glide mechanisms of dislocations. However, since these 2D models are based on the idealization of infinite dislocation lines, a number of important mechanisms and dislocation interactions are either not included into these models or accounted for in an implicit manner at best. These mechanisms include, crossslip, junctions, jogs, multiplication by Frank-Read sources, and line tension associated with self- 
energy. These difficulties have been addressed in a pioneering three-dimensional dislocation model which was developed by Kubin and his co-workers [10] and Canova, et al. [11]. Their model was based on the discretization of dislocation curves into succession of pure edge and pure screw dislocation segments of fundamental length, corresponding to the discretized lattice. More recently, a new approach for 3D dislocation dynamics has been established by Zbib, Hirth and Rhee [12-14]. In this approach arbitrarily curved dislocations are decomposed into piecewise continuous arrays of mixed straight segments in a continuum crystal and long range interactions are treated using super-dislocations, allowing for the treatment of large dislocation densities.

The 3D discrete dislocation model (micr3d) developed at WSU simulates the dynamical behavior of large numbers of dislocations of arbitrary shapes, interaction among groups of $3 \mathrm{D}$ dislocations and, therefore, the behavior of prescribed cell walls. In the model there are a number of rules and models for short-range reactions which, in turn, have a decisive effect on the predicted hardening and evolution of the structure. These issues have been, recently, addressed by Rhee et al. [13] who developed numerical rules for implementation into DD models to treat short-range interactions, by introducing a critical force criterion. In this paper, we investigate these reactions and corresponding mechanisms that contribute critically to work hardening during deformation using the dislocation dynamics model. The main issues we address are junction formation and strength through the process of "zipping" and "unzipping", jog formation and strength, and dipole strength. It is shown that the full dynamics of these interactions can be explicitly captured via dislocation dynamics simulations, where the dynamics of these configurations is determincd by direct calculation of driving forces and internal stresses. The simulation model is then utilized to provide possible explanations of irradiation induced hardening in metals subjected to cascade damage.

\section{BASIC EQUATIONS}

The complete description of the 3D dislocation model (DD) can be found in [12-14]. Here we give a brief outline of the main features of the model and basic governing equations. The main problem is that of nonlinear interaction of large number of curved dislocations of arbitrary shapes. The crystal is treated as a continuum with dislocations restricted to move on 
crystallographic slip systems. The model has been developed for both fec and bec single crystals. For bcc single crystals we consider the $\{110\}<111>$ and $\{112\}<111>$ slip systems which are the most close packed slip systems and both are active at low temperatures. The $\{123\}<111>$ slip systems are less close packed and become active at high temperatures. For fcc single crystals, $\{111\}<011>$ slip systems, and hence glissile dislocation lines can only lie on $\{111\}$ planes.

Each plane contains a number of dislocation curves and loops whose configurations are approximated by a series of straight scgments of mixed character as illustrated in Figure 1a. There are a number of advantages in using mixed segments of arbitrary lengths and orientation:

1. Segment length depends upon the local curvature. Dislocation curves with small curvature are segmented with long segments, while those with large curvatures are meshed with shorter segments (typical segment size could vary from $50 b$ to a few hundred $b$, where $b$ is the Burgers vector).

2. With mixed segments of arbitrary length and orientations, dislocation interactions such as junctions with any arbitrary configuration can be formed.

3. Mobility and strength of dislocation reactions, such as jog motion through dislocation bowout at the jog and junction destruction by the process of "unzipping" can be readily captured.

4. The stress field of arbitrary mixed straight dislocation segment in an isotropic medium is given in a closed form.

\subsection{Long Range Interaction and Self-Force}

The stress field of a finite dislocation segment is given by Hirth and Lothe [15] and deWit [16]. The interaction force per unit length (Peach-Koehler force " $P K$ force") a given segment exerts on a remote segment is evaluated at the center of the remote segment. This approximation is valid for two segments that are far apart from each other since the variation of the interaction force along the segment length is very small. For adjacent segments forming a bend the interaction force and self-force varies significantly along the dislocation line; it is singular at the bend and decays as $1 / r$. Therefore, we treat this case in a more rigorous way as described by 
Zbib et al. [12]. The solution for the total interaction force between the two adjacent segments shown in Figure $2 b$ is developed along the same lines described by Hirth and Lothe [15] for a dislocation bent with the same Burgers vector. The two segments could belong to the same dislocation line with the same Burgers vector or they could be at a junction node with two different Burgers vector. The result is an average glide force per unit length given by

$$
\begin{gathered}
\boldsymbol{F}_{2}=\frac{\mu}{4 \pi} \ln \frac{t}{\rho} \sqrt{[} \boldsymbol{b}_{z 1} \boldsymbol{b}_{z 2} \frac{\cos \theta-1}{\sin \theta}-\boldsymbol{b}_{z 2}^{2} \frac{v \sin \theta \cos \theta}{1-v}+\boldsymbol{b}_{x 1} \boldsymbol{b}_{z 2} \frac{v}{1-v}+\boldsymbol{b}_{x 2} \boldsymbol{b}_{z 2} \frac{v}{1-v}\left(2 \sin ^{2} \theta-1\right) \\
\left.+\boldsymbol{b}_{x 1} \boldsymbol{b}_{x 2} \frac{\cos \theta-1}{(1-v) \sin \theta}+\boldsymbol{b}_{x 2}^{2} \frac{v \sin \theta \cos \theta}{1-v}+b_{y 1} b_{y 2} \frac{\cos \theta-1}{(1-v) \sin \theta}\right]
\end{gathered}
$$

The normal force component out of page is given by

$$
\begin{aligned}
\boldsymbol{F}_{\boldsymbol{n}}=\frac{\mu}{4 \pi(1-v)} & \ln \frac{ \pm}{\rho} \sqrt{[} \boldsymbol{b}_{\boldsymbol{y} 1} \boldsymbol{b}_{x 2} \frac{v \sin \theta+\cos \theta-1}{\sin \theta}+\boldsymbol{b}_{x 1} \boldsymbol{b}_{y 2} \frac{\cos \theta-\cos ^{2} \theta-v \sin ^{2} \theta}{\sin \theta} \\
& +\boldsymbol{b}_{z 1} \boldsymbol{b}_{\boldsymbol{y} 2}(1-v)(1-\cos \theta)+\boldsymbol{b}_{y 1} \boldsymbol{b}_{z 2} v(2-\cos \theta) \\
& \left.-\boldsymbol{b}_{z 2} \boldsymbol{b}_{\boldsymbol{y} 2} v \cos \theta+\boldsymbol{b}_{x 2} \boldsymbol{b}_{y 2} \cos \theta+\boldsymbol{b}_{x 2} \boldsymbol{b}_{\boldsymbol{y} 2} v \sin \theta\right]
\end{aligned}
$$

Here $\mu$ is the elastic shear modulus, $v$ is the Poisson's ration, $L$ is the dislocation segment, and $\rho$ is the core parameter. For the case of general bend of two segments only (ignore segment " 3 : in Figure 2a) $\boldsymbol{b}_{1}=\boldsymbol{b}_{2}$ and equations (1) and (2) reduce to the expressions given in [15]. In passing we emphasize that equation (1) accounts explicitly for the self-force which gives rise to the so call "line tension".

\subsection{Dislocation Mobility}

The motion of each dislocation segment is determined by first evaluating the total $P K$ force which arises from all other dislocation stress fields and the applied stress, such that 


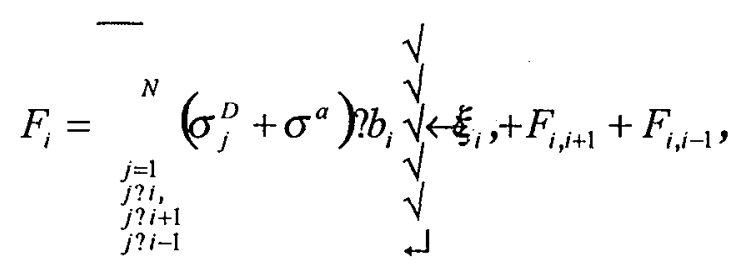

where $N$ is the total number of dislocation segments, $\sigma_{j}^{D}$ is the stress tensor from a remote segment $j, \sigma^{a}$ is the applied stress tensor, $\xi_{i}$ is the line sense vector, and $\boldsymbol{F}_{i, i+1}$ and $\boldsymbol{F}_{i, i-1}$ are the interaction forces between segments $i$ and $i+1$, and $i$ and $i-1$, respectively, as computed from equations (1) and (2). The effective shear stress $\tau_{e i}$ on segment $i$ is given by

$$
\tau_{e i}=\frac{f_{g i}}{b}-\sigma_{f}
$$

where $f_{g i}=\left|F_{i} ? \hat{v}_{i}\right|$ is the magnitude of the glide force per unit length with $\hat{v}_{i}$ being a unit vector in the direction of slip, and $\sigma_{f}$ is the friction stress arising from lattice damping effects. The velocity vector of segment $i$ is given by $v_{i}=v_{g i} \hat{v}_{i}$ where the basic relation for $v_{g i}$ is discussed below.

The relationship between the glide velocity and the glide force per unit length (or effective shear stress) is temperature-dependent. At high temperatures, dislocations (pure edge, pure screw, and mixed) move by the phonon drag mechanism and the driving effective force is athermal. There are a number of relations for the dislocation glide velocity $v_{g}$, including relations of power law forms and forms with an activation term in an exponential or as the argument of a sinh form. Often, the simple power law form is adopted for expedience, e. $g$. $v_{g}=v_{s}\left(\tau_{e} / \tau_{s}\right)^{m}$. In a number of cases of pure phonon/electron damping control or of glide over the Peierls barrier a linear form of equation (1), $m=1$, predicts the results very well. The linear form has been theoretically predicted for a number of cases as reviewed by Hirth and Lothe [15] leading to 


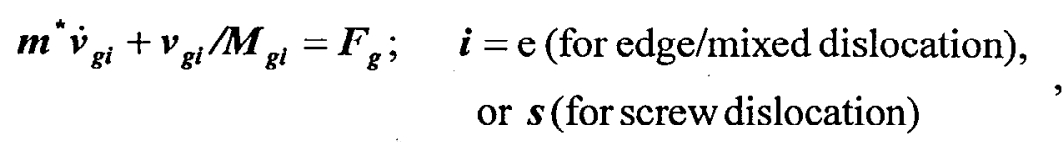

where $m^{*}$ is the effective mass and $M_{g i}$ is the dislocation mobility and, in general, it dependence on the character of the dislocation. Generally, $M_{g i}$ could be, among other things, a fuction of the angle between the Burgers vector and the dislocation line sense, especially at low temperatures. In bcc single crystals, at low temperatures a pure screw dislocation has a rather complex threedimensional core structure, resulting in a high Peierls stress which is overcome by stress-assisted thermal activation [12]. This leads to a relatively low mobility for screw dislocations while the mobility of mixed dislocations is very high [17].

In a more general treatment, the inertia term should be included in equation (6). However, recent studies have shown that the rise time for a dislocation to reach a steady state is on the order of $10^{-10} \mathrm{~s}$ for a dislocation velocity below 0.4 times the shear-wave velocity [18]. A typical time step in computer simulation is of the same order, making it possible to neglect the inertia term, especially for low mobility dislocations in bec metals. This inertia term, however, plays an important role for fast moving dislocations in fcc metals where the mobility is a few orders of magnitude higher than that in bec.

The result of the above formulation is a set of nonlinear first-order differential equations governing the motion of the dislocation segments. The motion of the dislocations gives rise to plastic strain $\boldsymbol{D}^{\mathrm{P}}$ and spin $\boldsymbol{W}^{\mathrm{P}}$ which are calculated from

$$
\mathbf{D}^{p}=\frac{-1}{\mathrm{~V}}_{\mathrm{i}=1}^{N}\left[\left(\mathbf{v}_{g i} \leftarrow \xi_{i}\right) \quad \mathbf{b}_{i} \ell_{i}\right]_{\mathrm{sym}}, \quad \text { and } \quad \mathbf{W}^{p}=\frac{-1}{\mathrm{~V}}_{\mathrm{i}=1}^{N}\left[\left(\mathbf{v}_{g i} \leftarrow \xi_{i}\right) \quad \mathbf{b}_{i} \ell_{i}\right]_{\text {anti-sym. }}
$$

where $\ell_{\mathrm{i}}$ is the segment length, $\mathbf{v}_{\mathrm{gi}}$ is the segment, $\mathbf{b}_{\mathrm{i}}$ is the Burgers vector, $\xi_{\mathrm{i}}$ is the sense vector, and $\mathrm{V}$ is the volume of the simulated crystal.

\subsection{Short Range Interactions}

For short-range interactions basic theories, which describe the underlying physical mechanisms at the core level, may be rigorously included into the 3DD model. Such an approach 
may be important when investigating the local interaction between two dislocations over small distances (close to the core). However, this approach is not desirable numerically or even important when dealing with relatively large numbers of dislocations on a large scale (10's of $\mu m$ ). It is more numerically efficient to develop rules based on a rigorous investigation of the two-dislocation interaction problem. Then, we implement these rules directly into the 3DD model for large-scale simulations. Therefore, in [13] we developed numerical rules for implementation into the 3DD model to treat short-range interactions. These rules are:

Rule 1: Critical force criterion " $F$ ? $F^{c}$ " for short-range interaction.

Rule 2: Critical force criterion for annihilation.

Rule 3: Critical-angle criterion for junction formation; $\theta_{A B} \leq \theta_{j n}^{c}$.

Rule 4: Critical-angle criterion for jog formation; $\theta_{A B} ? \theta_{j g}^{c}$.

Rule 5: Critical-angle criterion for jog strength; $\theta_{j g} \leq \theta_{j g s}^{c}$.

Here $\theta_{A B}$ is the angle between two dislocation segments, $\theta_{j g}$ is the bow-out angle at a jog, and $\theta_{j n}^{c}, \theta_{j g}^{c}$, and $\theta_{j g s}^{c}$ are the critical values for junction formation, jog formation and jog strength, respectively. Each of these rules involves a critical value that determines the interaction. We suggest that numerical values for $\theta_{j n}^{c}$ and $\theta_{j g}^{c}$ should be determined from a rigorous investigation of the two-dislocation interaction problem, similar to those performed by Huang et al [19] for the dipole problem. Values for the critical force, jog strength, as well as dislocation mobility maybe quantified explicitly by means of simulations at the core level, such as MD simulations [20]. These simulations could be very extensive since one would have to examine a number of possible combinations of Burgers vectors and slip planes.

\section{BASIC REACTIONS AND STRENGTHENING MECHANISMS}

(In the following discussion we consider the deformation of a single crystal Ta at room temperature for which $b=2.86 \leftrightarrow 10^{-10} m, \mu=70.7 G P a, v=0.339, \sigma_{f}=3 \leftrightarrow 0^{-5} \mu$ ). 


\subsection{Junction Formation - Zipping}

The process of dislocation junction formation has been addressed schematically by many authors, but no quantitative analysis has been performed except by the recent work of Bulatov et al. [21]. We study this phenomenon using the DD dislocation dynamics model. The process begins when two attractive dislocations gliding on intersecting planes (Figure 2a) meet at the line of intersection and combine if the reaction is favorable (Figure $2 \mathrm{~b}$ ). In the DD simulations this is captured through explicit evaluation of the system dynamics, i.e. there is no need to check if

Frank's rule (i.e. $\left|b_{1}+b_{2}\right|^{2}<\left|b_{1}\right|^{2}+\left|b_{2}\right|^{2}$ ) is met before combining the segments. If the two segments are attractive (Rule 1) and their energy would be at a minimum by forming a junction, they would approach each other, align themselves into a parallel configuration (Rule 3), react and form a junction. Once initiated, the junction extends along the line of intersection by the process of "zipping" as anticipated by the Friedel-Saada model [22] (Figure 2c). The configurations shown in Figures $2 \mathrm{a}-\mathrm{h}$ and $3 \mathrm{a}-\mathrm{b}$ are typical examples of junctions formed using DD simulations.

\subsection{Junction Destruction - "unzipping"}

The extension or destruction of the junction takes place by the motion of the dislocationnode at the triple point along the line of intersection as anticipated by the Freidel-Saada model[22] and the recent $\mathrm{MD}$ simulations of Bulatov et al. [21]. This process is captured by DD simulations as shown in Figures 2d-f and 3a,b. Thus, as also pointed out by Bulatov et al [21], the force driving nodal stacking-fault energy determines the strength and fate of the junction.

The process of junction formation and destruction is a balance between applied stress, dislocation interaction forces and core energy around the junction node. As the reversed stress increases to a critical value the forces on the dislocation nodes increase and move the nodes towards cach others along the line of intersection. In the simulation, once the junction is formed and reaches a stable configuration as shown in Fig. 4a, the applied stress is reversed incrementally. Due to the line tension effect, a higher stress is required to overcome the elastic interaction energy and core energy to unzip the junction. Figure $4 \mathrm{~b}$ shows the effect of line tension on the critical stress to unzip and break a junction. We can deduce from the figure that for a longer dislocation branch from the source $(L)$ less force is required to overcome the elastic 
energy barrier for the unzipping process to occur. This is due to the fact that the force associated with line tension is larger with larger " $L$ ", resulting in higher self-force at the junction node.

\subsection{Jog Strength}

Jogs can move by creating point defects such as vacancies or interstitials. The strength of these defects is balanced by the total line tension of two adjacent segments around a jog, leading to the following expression for the critical bow-out angle $\theta_{j g s}^{c}$ for jog to move [14]

$$
\cos \frac{\theta_{j g s}^{c}}{2} \sqrt[V]{ }=\frac{W}{\mu b^{3}}
$$

where $W$ is the interstitial or vacancy formation energy. To investigate the effect of the critical jog angle on the corresponding critical stress for jog motion, we carried out DD simulations for the configuration shown in Figure 5. The result is obtained by considering a simple Frank--Read source with a jog located in the middle. For a given $\theta_{j g s}^{c}$ the stress is increased incrementally until the jog begins to move. Figure $4 a$ shows a snapshot of the simulation result after the jog has moved from its initial position. As the critical angle decreases, higher stress is required to move the jog as shown in Fig. 5c. However, further decrease in the angle (less than 60 degrees) does not result in a significant increase in the critical stress. This is because once the bow out reaches the unstable configuration as in the Frank--Read source, less stress is required for the dislocation to wrap around the source. In the absence of a jog, the critical stress required to bowout a Frank--Read source of length $6000 \mathrm{~b}$ or $3000 \mathrm{~b}$ is $11 \mathrm{MPa}$ or $23 \mathrm{MPa}$ respectively. This value is, indeed, the minimum threshold for the critical stress to bowout the Frank--Read source with a very weak jog, corresponding to a critical jog angle approaching 180 degrees.

\subsection{Dipole Strength}

Although models describing the dipole strength are available, the dipole is assumed to be infinitely long. In our approach, dipole brcak occurs naturally if the stress requircd to cause it to unzip apart is sufficient. Consider, for example, the dipole which is formed by the bow-out of dislocations from Frank-Read sources shown in Fig. 6a. The strength of the dipole depends on 
the normal separation $h$ and the length of the distance from dipole position to the dislocation endpoint $d$ where the dislocations cmanate.

Figure $6 \mathrm{~b}$ shows the critical stress to unzip a dipole as a function of the normal separation and the separation between the original sources. For a constant dipole separation $h$, dipole with a smaller value $d$ requires higher stress to unzip it than the case with larger distance. This is due to the line tension from the dipole to the pinning point. For larger distance of $d$ the curvature of the dislocation at the dipole position is larger. This, in turn, results into a larger line tension, making it easier to unzip the dipole, similarly to the case of junction unzipping. Furthermore, we note that, due to the line tension, the critical shear stress to break the dipole is much smaller that that predicted for the case of two infinite dislocation segments which is given by

$$
\tau_{c}=\frac{\mu b}{8 \pi(1-v)} \frac{1}{d}
$$

\section{DOUBLE SLIP DEFORMATION}

In this section we present typical results of the deformation of single crystals. Figure 6 shows a cubic cell of size $10 \mu \mathrm{m}$. In this case, the material is simulated is single crystal Ta whose properties are given in Section 3. The crystal contains 50 Frank-Read source distributed randomly on the two slip planes (110) and $(\overline{1} 10)$ as shown in Figure 6 . The size of the dislocation sources varies from $5000 \mathrm{~b}$ to $8000 \mathrm{~b}$. This gives an initial dislocation density of $10^{11} /$ $\mathrm{m}^{2}$. The load is applied in the [100] direction with a constant strain rate of $10 / \mathrm{s}$. The resolved shear stresses on both planes are equal, activating both slip systems. The mobility of edge and general dislocations is $10^{2} /(\mathrm{Pa} . \mathrm{s})$ and that of screw character is assumed to be smaller by one order of magnitude.

A snapshot of the dislocation structure after 163,000 iterations is shown in Figure 7 . As a result of dislocation intersections, many jogs and junctions form and their total number increases with further deformation (the total number of jogs in Fig. 7 is 184). The simulation is performed for a jog critical angle (strength) of 120 degrees. A typical stress--strain curve is shown in Figure 
8. We can be deduce from the figure that significant strain hardening occurs as a result of formation of jogs and functions. The dislocation density in Figure 7 is $4.3 \times 10^{11} / \mathrm{m}^{2}$.

Figure 9 shows results obtained for a single crystal Molybdenum deformed at room temperture. The size of the simulation cell is $30 \mu \mathrm{m}$. For Mo: $\boldsymbol{b}=2.725 \leftrightarrow 40^{-10} \mathrm{~m}, \mu=123 \boldsymbol{G P a}$, $v=0.305, \sigma_{f}=3 \leftarrow 10^{-5} \mu$. The mobility of edge and general dislocations is $10^{3} /(\mathrm{Pa}$. s) and that of screw character is assumed to be $25 /$ (Pa.s). This estimate is based on the experimental results of Prekel and Conrad [23]. The low mobility of the screw segment results in a dislocation structure dominated by extended screw dislocations consistent with typical TEM results [23].

\section{Irradiation Induced Hardening}

Finally, we consider the problem of irradiation induced hardening problem in alloys subjected to cascade damage. In order to illustrate the possibility of the DD simulation in providing rigorous understanding of this phenomenon, we consider the defect structure in $\mathrm{Cu}$. The structure consists of prismatic dislocation loops resulting from collapse of vacancies or interstitials [24-29]. The main issue we look into is the phenomenon of increased yield stress resulting from radiation. The subsequent phenomena of yield drop and localized deformation will be investigated later.

As pointed out by Trinkaus et al. [28], the irradiated induced hardening cannot be rationalized in terms of conventional dispersed hardening. However, this phenomenon may be understood in terms of cascade induced source hardening in which the dislocations are considered to be locked by the loops decorating them. Figurc 10a shows a dislocation decorated by loops as described in [28]. For an infinite dislocation, the critical stress to unlock the dislocation is approximated by [28] as

$$
\sigma_{y} \cong 0.069 \frac{\mu}{1-v} \frac{b-d}{L} \frac{y^{2}}{y}
$$

For $\mathrm{Cu}$ with $\mu=55 \mathrm{GPa}, v=1 / 3, L=200 \mathrm{~b}, d / y=3 / 2$, equation (10) yield $\sigma_{y}=61 \mathrm{MPa}$. However, when we consider a finite dislocation pinned at both ends as in Frank-Read Source, the 
critical stress required to unlock the dislocation will depend upon the dislocation length as well as the loop structure. This is shown using the DD simulation and the result is given in Figures $10 \mathrm{c}$ and 10d. In Figure 10c the dislocation becomes unstable when the stress reaches $100 \mathrm{MPa}$. In this case the dislocation is not decorated by loops. However, when the dislocation is decorated with loops the yield stress increases to $130 \mathrm{MPa}$. The reason the stress obtained from the DD simulation is higher that that obtained by the simple model is due to the line tension.

The results just presented illustrate the basic mechanism of hardening as captured by the DD simulation. Full analyses with large dislocation densities and various distributions are currently under way. Typical results are shown in Figures 11 and 12. In Figure 11 we show a periodic distribution of loops decorating dislocations with a loop density of $10^{19} / \mathrm{m}^{3}$. For random distribution of loops, the dislocation percolates around the obstacles as shown by the DD simulation given in Figure 12. Typical predicted stress-strain curve is shown in Figure 13. Detailed analysis along these lines are now underway and will examine the effect of radiation dose (loop density) on the initial yield stress, yield drop and localization.

\section{CONCLUSIONS}

Basic dislocation mechanisms, which contribute to yielding and strain hardening in metals, have been investigated using dislocation dynamics. These mechanisms include, dipole, dislocation intersections (junctions and jogs formation), and dislocation-defect interaction (cascade damage). These mechanisms are studied according to DD rules and models that have been developed to treat short-range reactions in bec and fcc metals. We have shown that these mechanisms have on the overall stress-strain behavior of metals has been illustrated. The result suggests that the DD simulation model provide a very valuable tool for investigating critical dislocation phenomena that control plastic deformation and hardening in metals. This type of microscopic modeling of deformation provides a natural transition from the atomic scale to the continuum crystal scale. It is suggested that, while MD simulations may provide needed rules of dislocation dynamics and interaction based on fundamental principles, DD simulations provide the most rigorous means for transmitting the influence of these critical mechanisms from the dislocation length scale to the macroscopic scale. 


\section{ACKNOWLEDGMENTS}

The support of the Lawrence I ivermore National I aboratory under contract No. W-7405-ENG48 with the DOE, is gratefully acknowledged.

\section{REFERENCES}

1. S. Mader, A. Seeger and H.M. Thieringer, J. Appl. Phys. 34, p. 3376-3386 (1963).

2. H. Mughrabi, Mater.Sci.Engng, 85, p. 15--31 (1987).

3. M.S. Whelanin, in: The Physics of Metals, edited by P.B. Hirsch, Part 2: Defects, Cambridge Univ. Press, p. 98 (1975).

4. U. Essmann and H. Mughrabi, Phil. Mag. A, 40, No. 6, p.731-756 (1979).

5. P.J. Woods, Phil. Mag. 28, p. 155-191 (1973).

6. N., Hansen and D. Kuhlmann-Wilsdorf, Matl. Sci. Eng., 81 p 141 (1986).

7. D., Kuhlmann-Wilsdorf, Mat. Res. Innovat, 1, p. 265 (1989).

8. D. Holt, J. Appl. Phys., 41, 3197-3201 (1970).

9. E.C. Aifantis, Sol. Stat. Phenom., 3 \& 4, 397-406 (1988).

10. L. P. Kubin, Phys. Stat. Sol (a), 135, 433-443 (1993).

11. G. R. Canova Y. Brechet and L.P. Kubin, in: Modelling of Plastic Deformation and Its Engineering Applications, edited by S.I. Anderson et al, Riso National Laboratory, Roskilde, Denmark. (1992).

12. H. M. Zbib, M. Rhee and J.P. Hirth, Int. J. Mech. Science, 40, p. 113-127 (1998).

13. M. Rhee, H.M. Zbib and J.P. Hirth, J.P., Modeling \& Simulations in Mater. Sci. \& Engr, 6, p. 467-492 (1998).

14. J.P. Hirth, M. Rhee and H.M. Zbib, J. Computer-Aided Materials Design, 3, p. 164-166, (1996).

15. J.P. Hirth and J. Lothe, J., Theory of Dislocations, Wiley, NY. (1982).

16. R. de Wit, Phys. Stat. Sol., 20, p. 567 (1967).

17. N. Urabe N. and J. Weertman, Mater. Sci. Eng., 18, 41 (1975).

18. J.P. Hirth, H.M. Zbib and J. Lothe, Modeling \& Simulations in Maters. Sci. \& Enger. 6, 165169 (1998). 
19. H. Huang, N. Ghoniem, T. Diaz de la Rubia, M. Rhee, H.M. Zbib and J.P. Hirth, to appear in ASME-JEMT, 1999.

20. S.J. Zhou, D.L. Preston, P.S. Lomdahl and D. M. Beazley, Science 279, p. 1525 (1998).

21. V. Bulatov, F. F. Abraham, L. Kubin, B. Devincre and S. Yip, Nature 392, p.669 (1998).

22. J. Friedel, Dislocations, Pergamon, Oxford (1964).

23. H.L. Prekel and H. Conrad, in: Dislocation Dynamics, ed. Rosenfield et al., McHraw-Hill (1967).

24. Fleischer, R.L., Acta Metll., 10, p. 835 (1962).

25. Hirsch, P.B., in :Vacancies 76, eds. Smallman, R.E., Harris, J.E., The Metals Society, p. 95. (1976)

26. Friedel, J., Dislocations, Oxford Press, p. 225 (1964).

27. Kocks, U.F., Phil. Mag., 13., p. 541 (1966).

28. Trinkaus, H., B.N. Singh, A.J.E. Foreman, J. Nuc. Materials, 251, p. 172, (1997).

29. Y. Dai , Theses No. 1388 (1995), Ecole Ploytechnique Federale de Laussanne. 

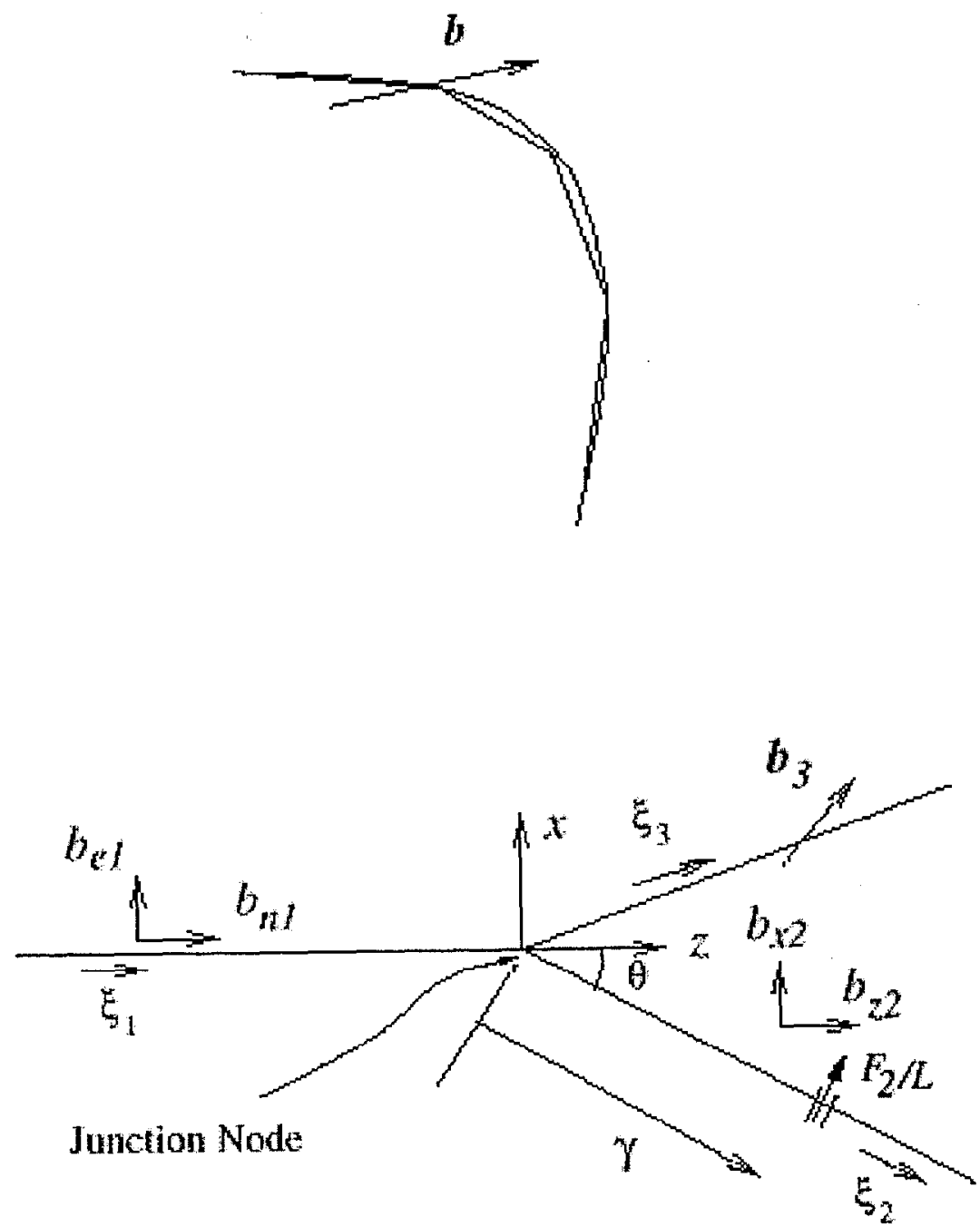

Figure 1. a) Discretization of a dislocation curve, b) Dislocation bend or junction. 
a)

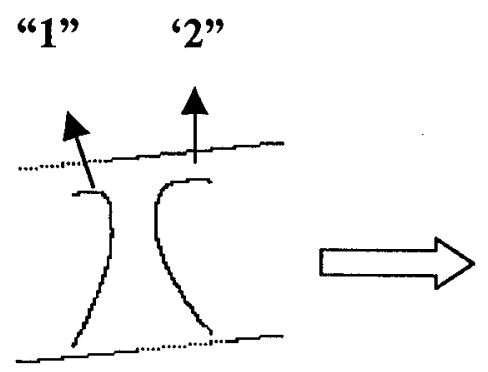

d)

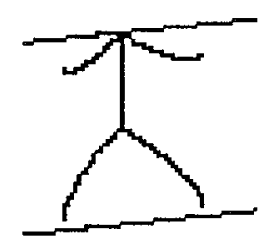

b) initiation

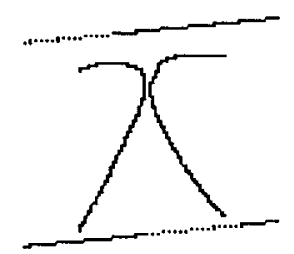

c) zipping
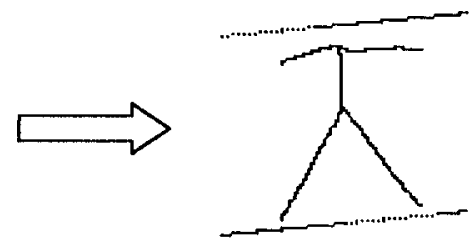

f) Breaking

e) unzipping

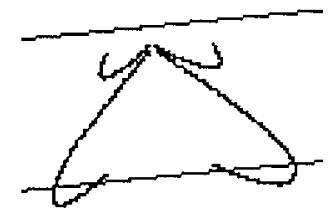

Figure 2. a-b) Junction formation "zipping" with stress of $20 \mathrm{MPa}$ applied in the [001] direction. d-f) Junction destruction "unzipping" with reversed stress (-30MPa).

Dislocation "1": $b / \sqrt{3}[\overline{1} 11](110) ;$ dislocation " $2 ": b / \sqrt{3}[11 \overline{1}](1 \overline{1} 0)$. Sessile junction is in the [001] direction with Burgers vector $2 b / \sqrt{3}[010]$. 
a)

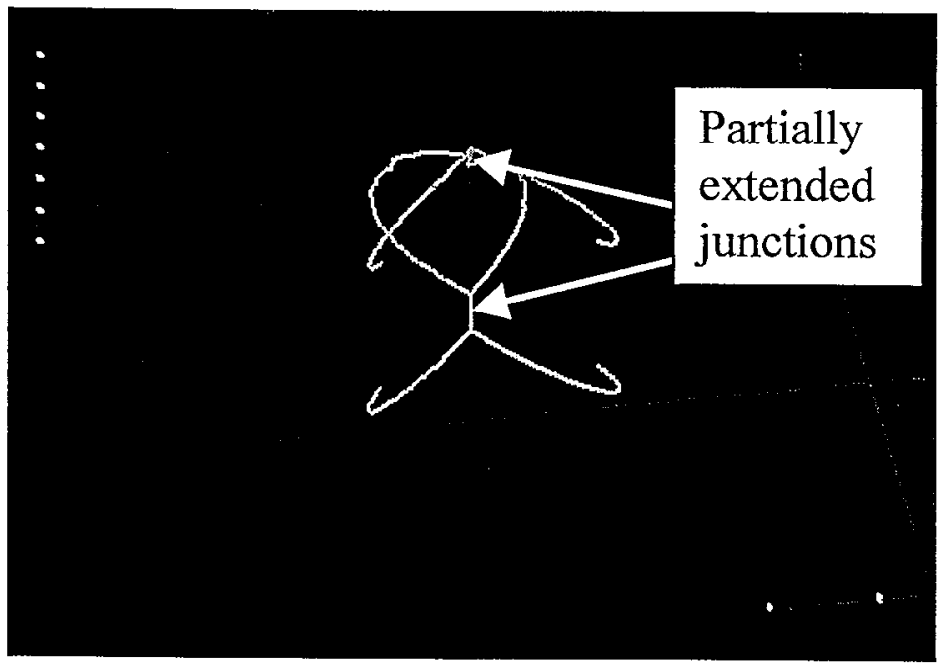

b)

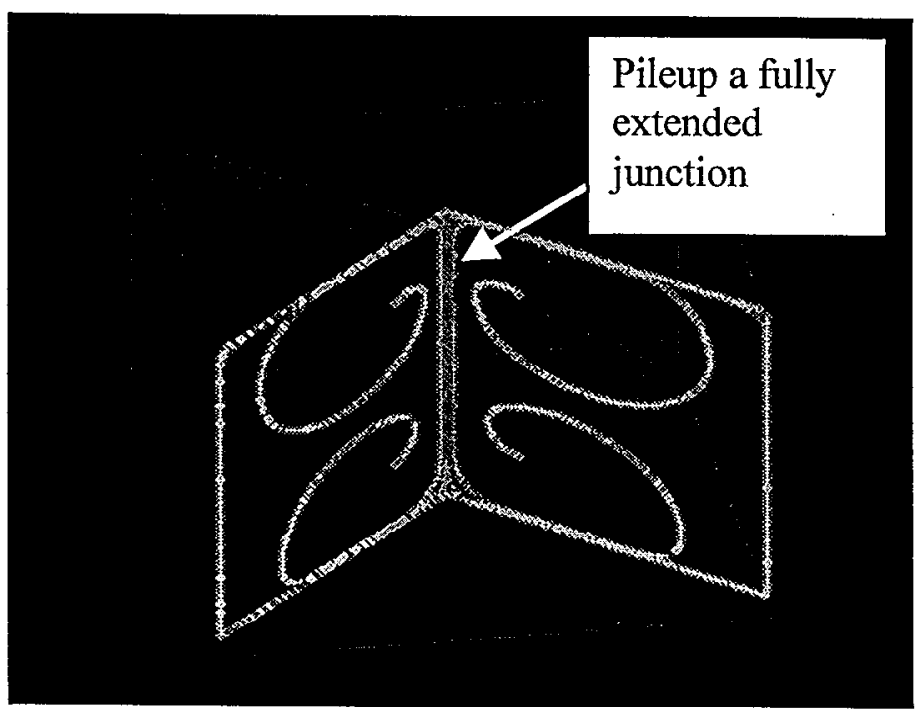

Figure 3. a) Formation of partial junctions and unzipping with stress increased to $50 \mathrm{MPa}$ (not reversed). b) A pileup of dislocations at a fully extended junction. 
a)
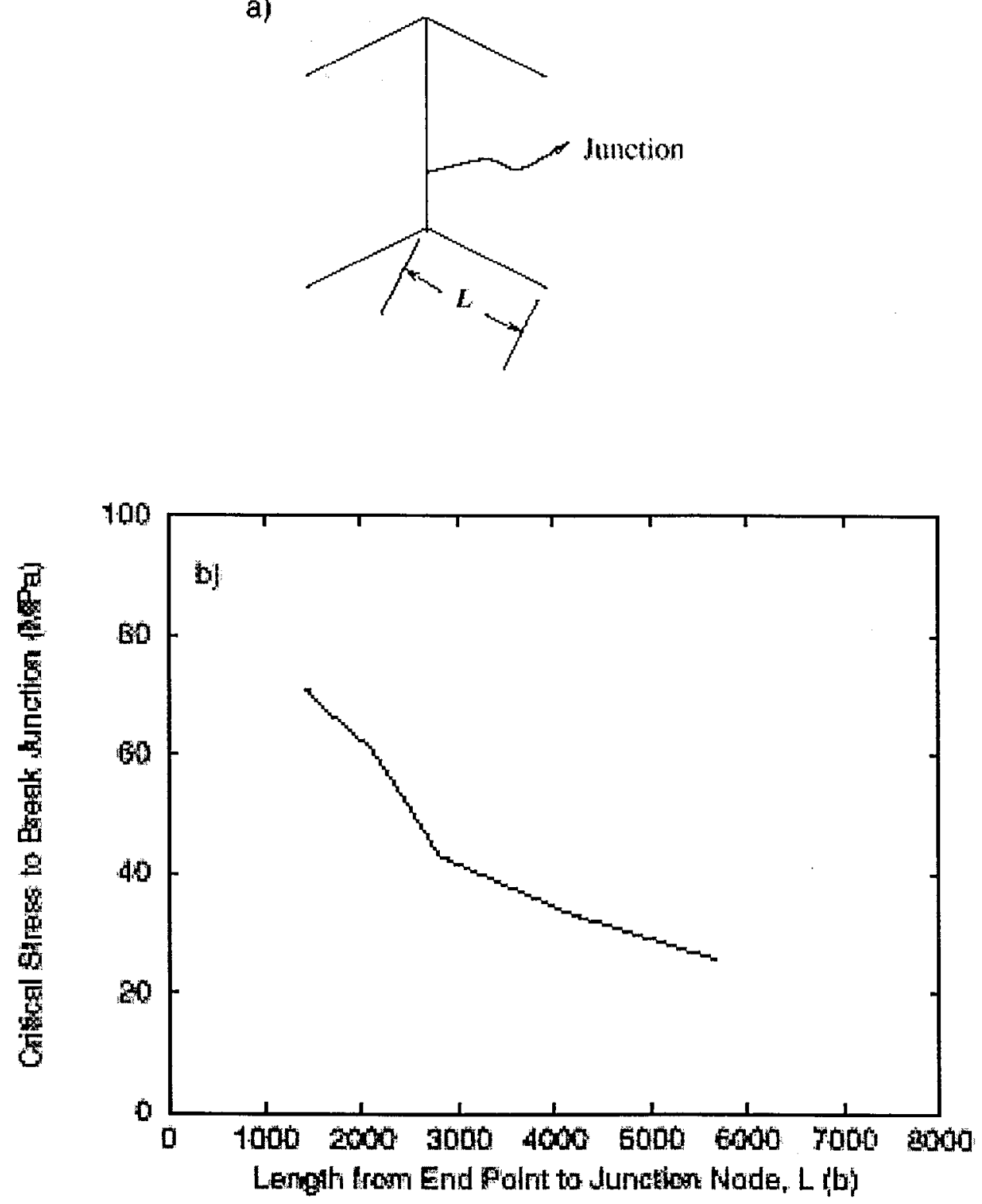

Figure 4. a) Fully extended junction formed as in Figures 2a-c, then the stress is reversed incrementally until the junction is completely destroyed as in Figure $2 \mathrm{f}$. b) The corresponding critical stress to break the junction as a function of " $\mathrm{L}$ " 
a)

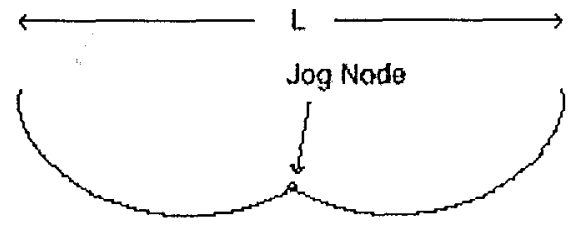

b)
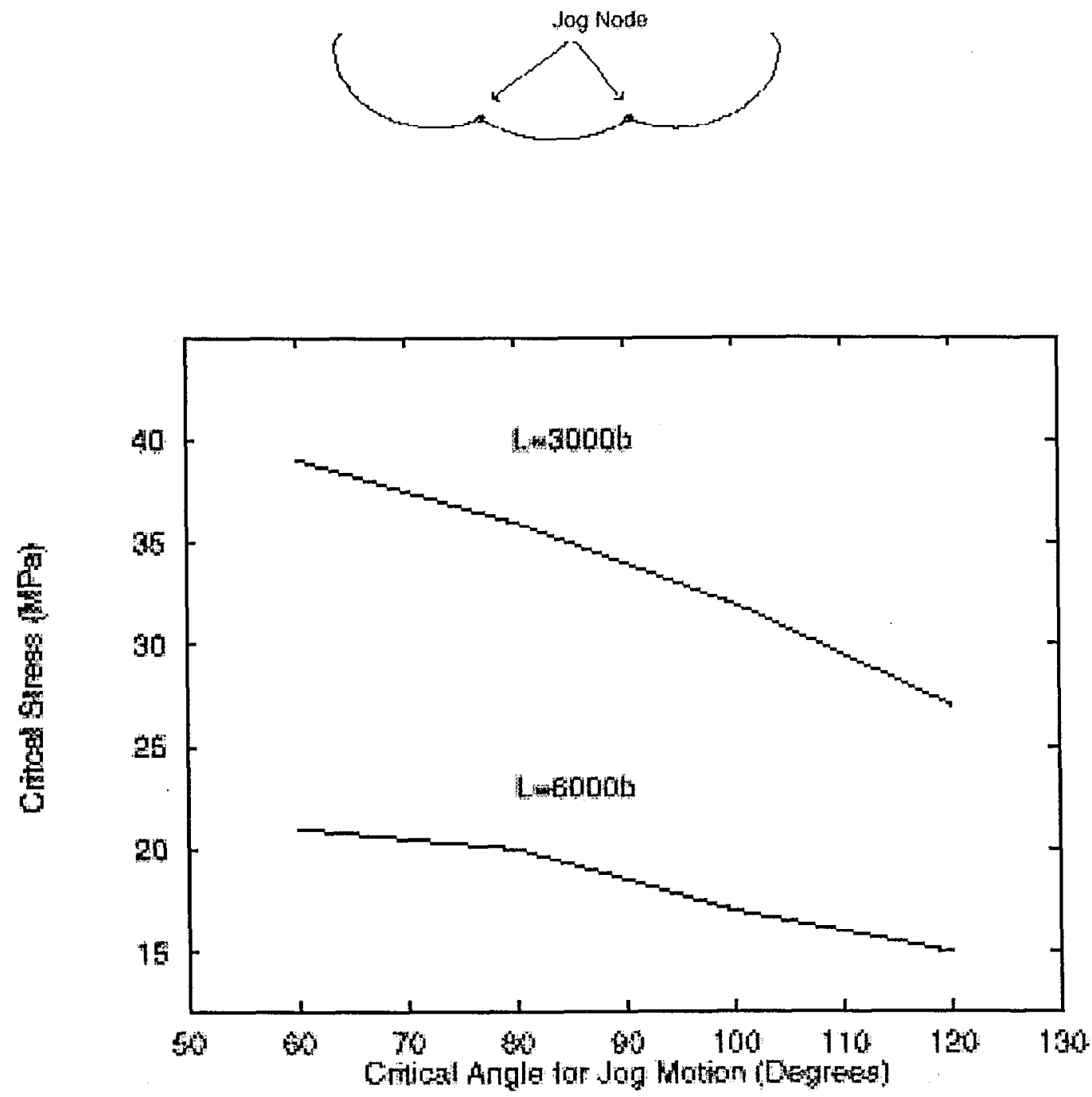

Figure 5. a,b) Pinned dislocations with jogs. c) Critical stress required to bow out and propagate the jogged dislocations as a function of jog critical angle (strength). 

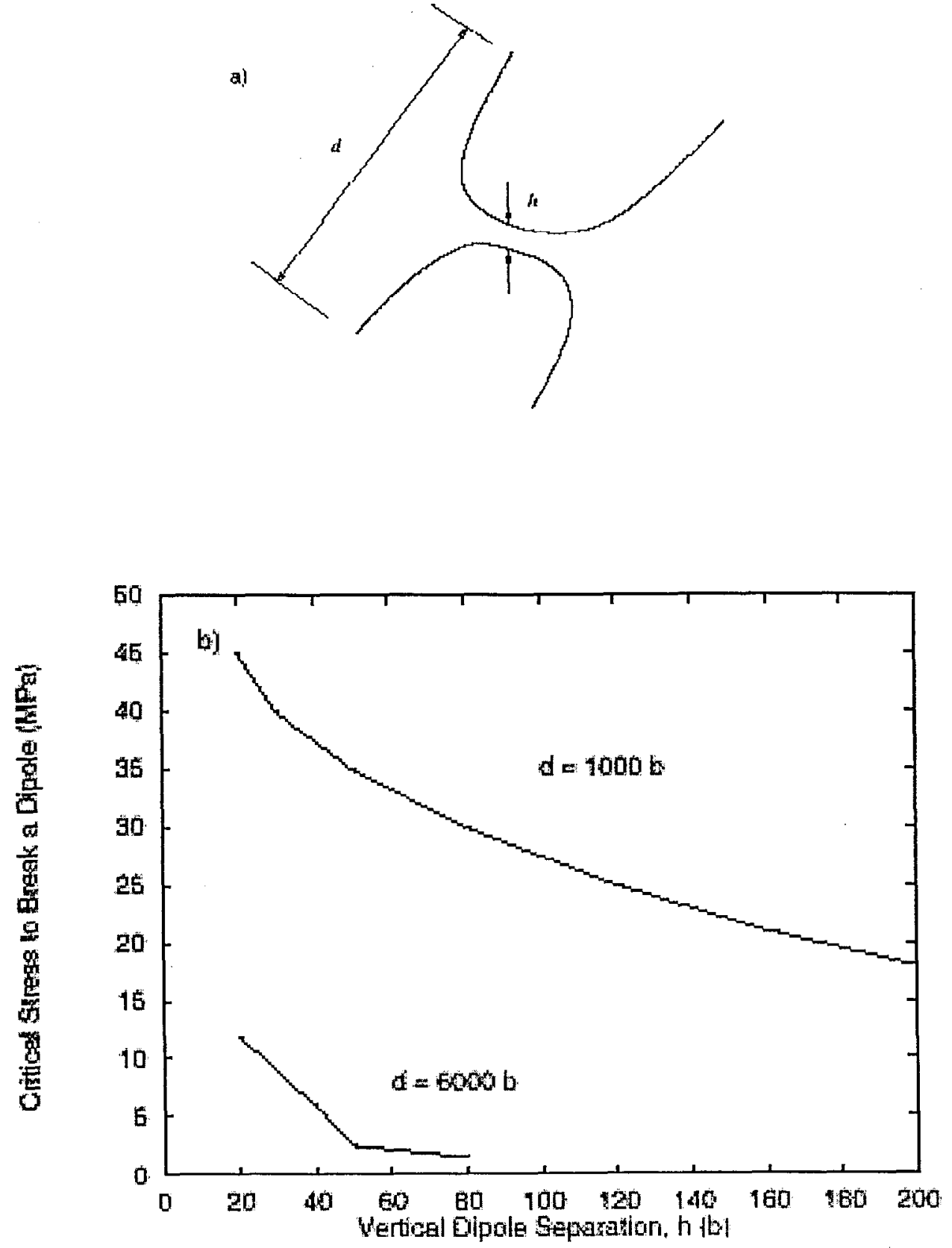

Figure 6. a) Two dislocations with opposite Burgers vectors emanating from two FrankRead sources on parallel planes separated by distance "h", forming a dipole "lock" at distancc $d / 2$. B) Critical stress to unzip the dipole. 
a)

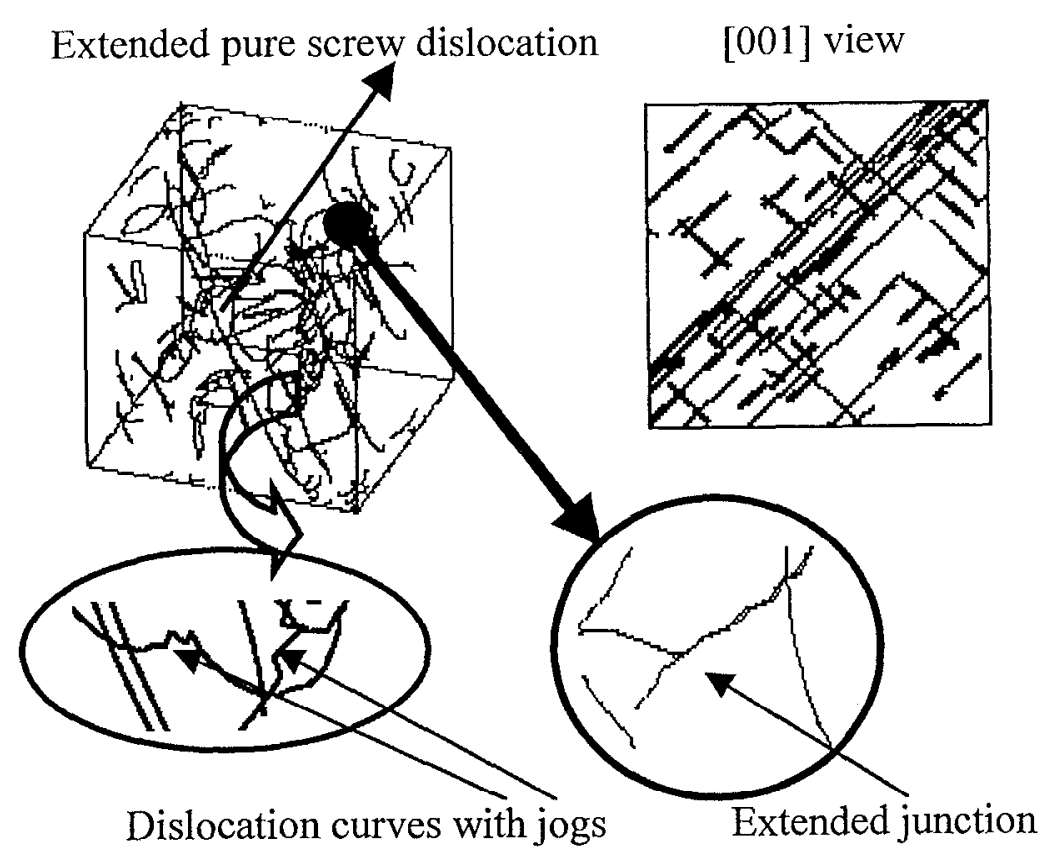

c)

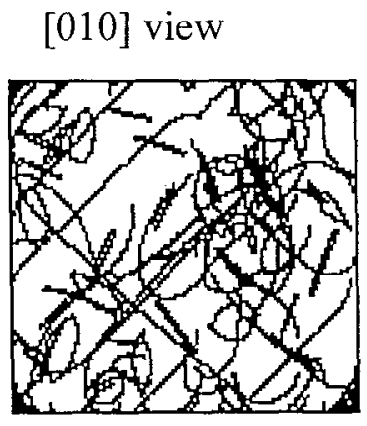

Figure 7. a) DD simulation of double slip deformation in Ta, b) [001] view, c) [010] view. Simulation cell size $=10 \mu m$ 


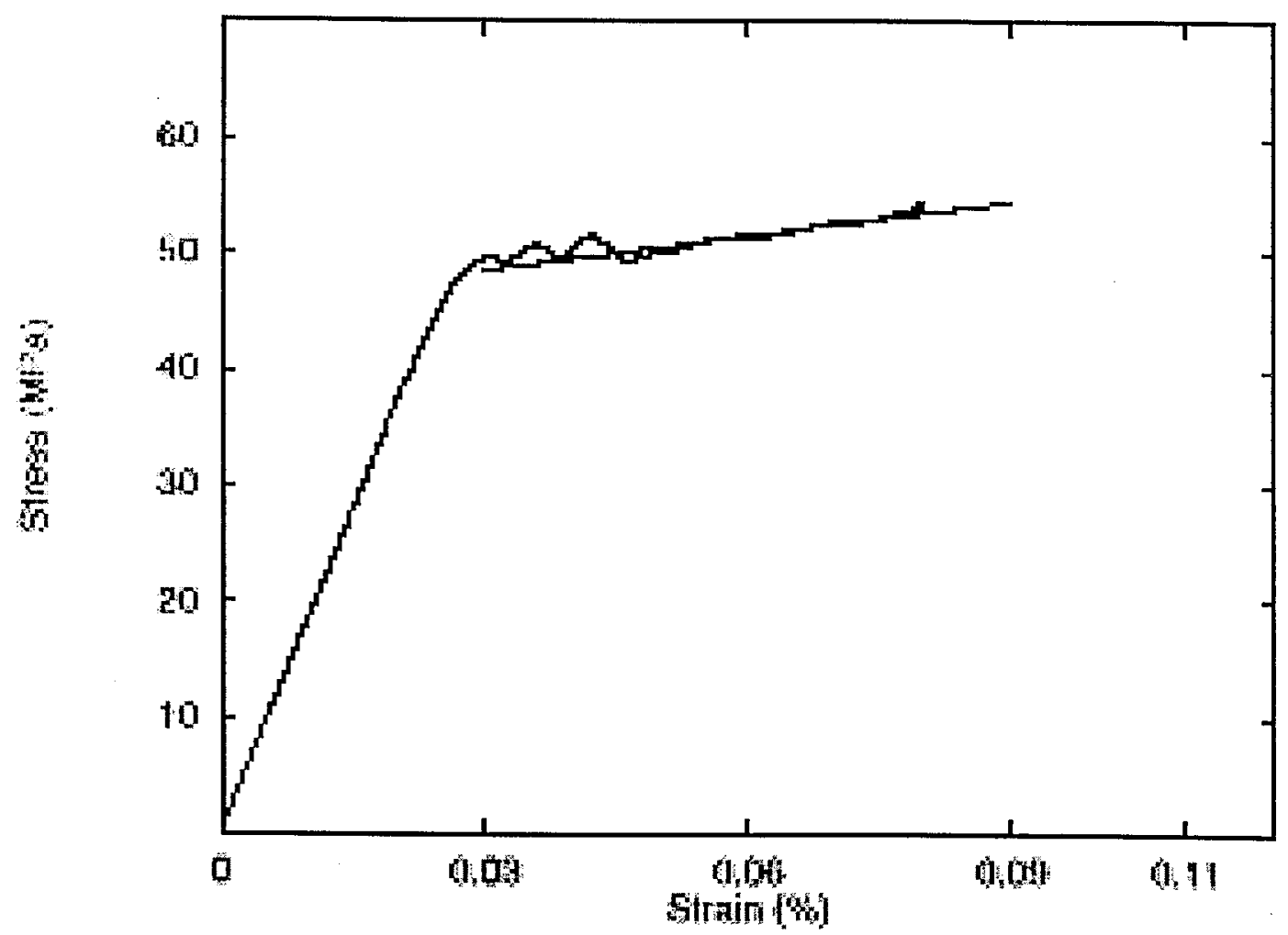

Figure 8. Stress-strain curve as predicted by the DD simulation of double slip deformation, resulting into the formation of jog and junctions and yielding strain hardening. 

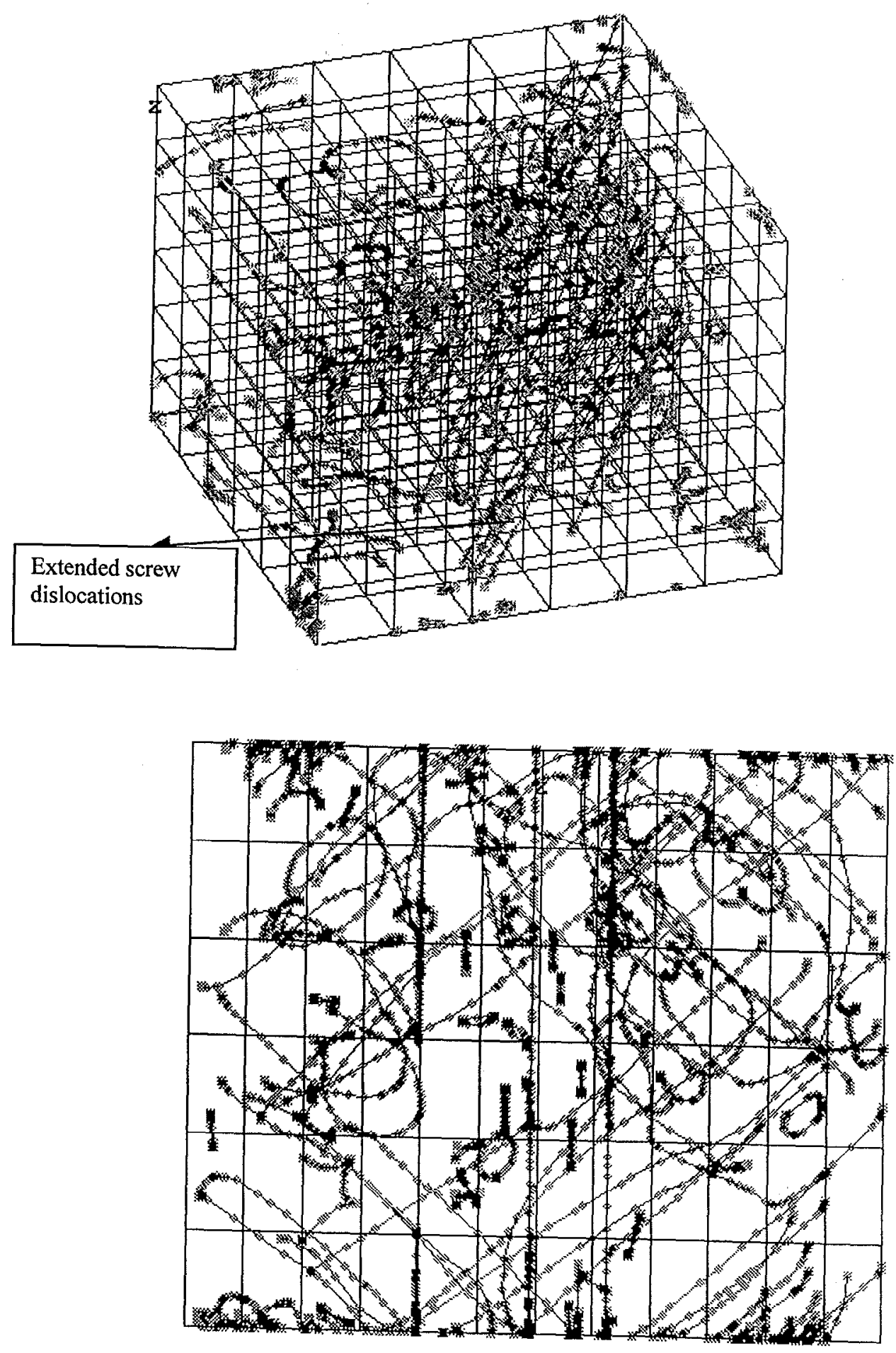

Figure 9. a) DD simulation of double slip deformation in Mo, b) [110] view. Simulation cell size $=30 \mu \mathrm{m}$. 
a)

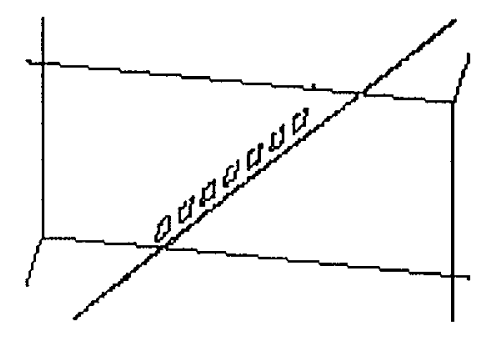

c)

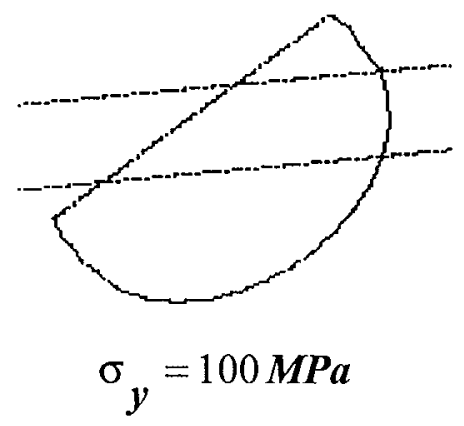

b)

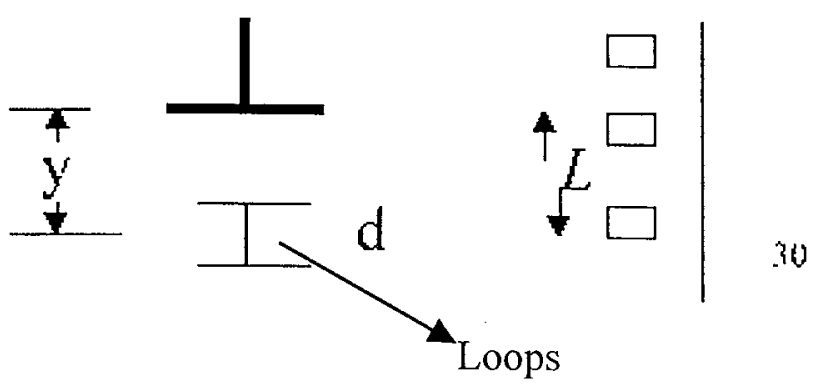

d)

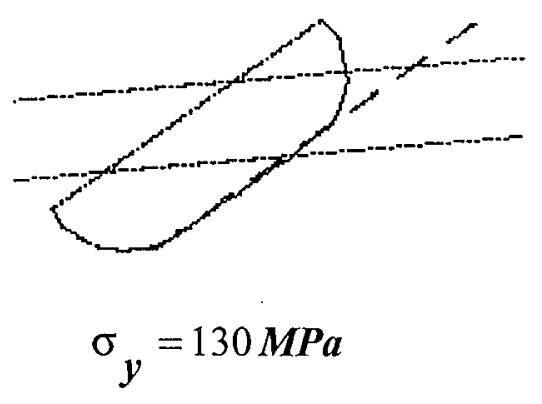

Figure 10. a) Prismatic dislocation loops decorating a mobile dislocation in $\mathrm{Cu}$. b) Side view showing ( $y=$ stand-off distance, $d=30 \mathrm{~b}$ is the loop size, $L=200 \mathrm{~b}$ ). c) Dislocation propagating from a Frank-Read Source and is not pinned by dislocation loops. d) Dislocation propagating from a Frank-Read Source and is pinned by dislocation loops. 


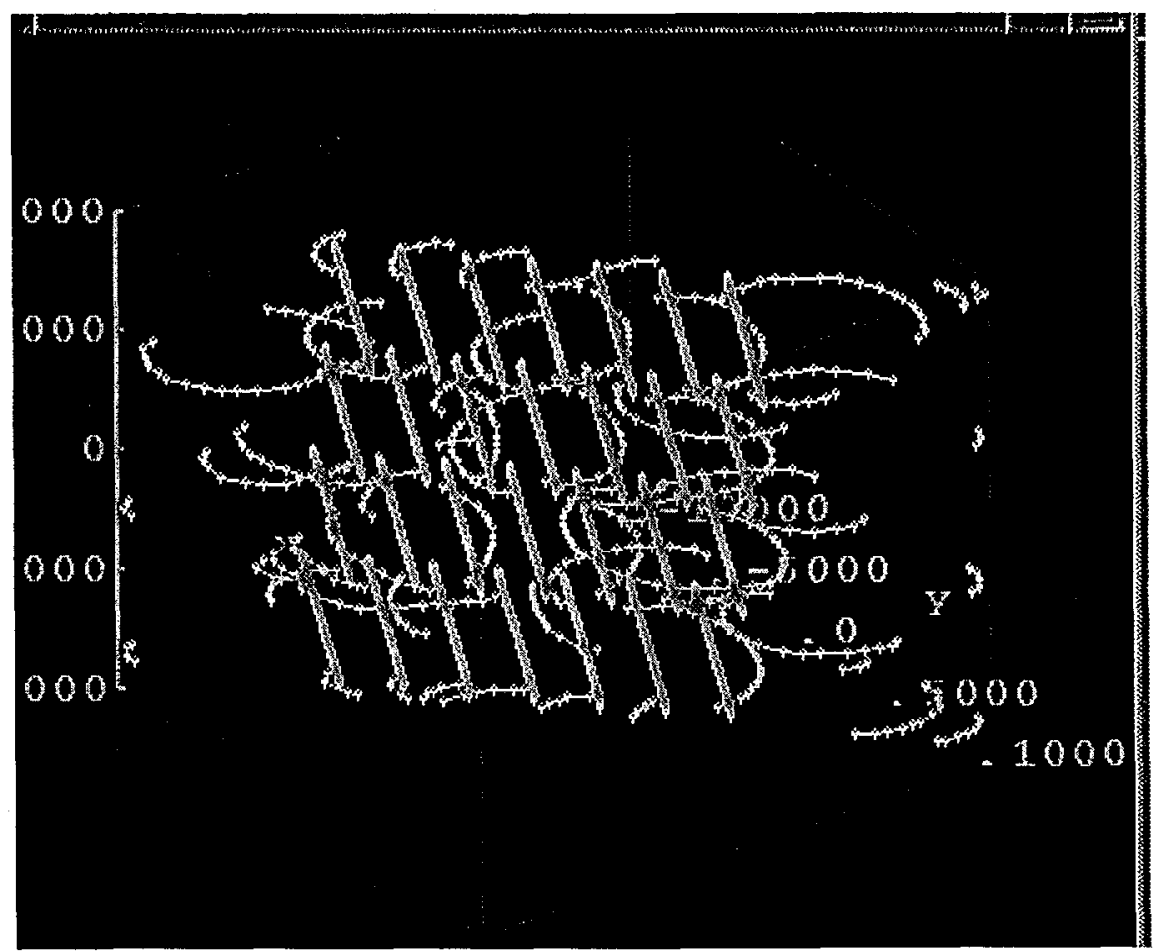

Figure 11. Simulation of dislocations decorated by dislocation loops in $\mathrm{Cu}$. Cell size $=10 \mu m$, loop density $=10^{19} / \mathbf{m}^{3}$. 


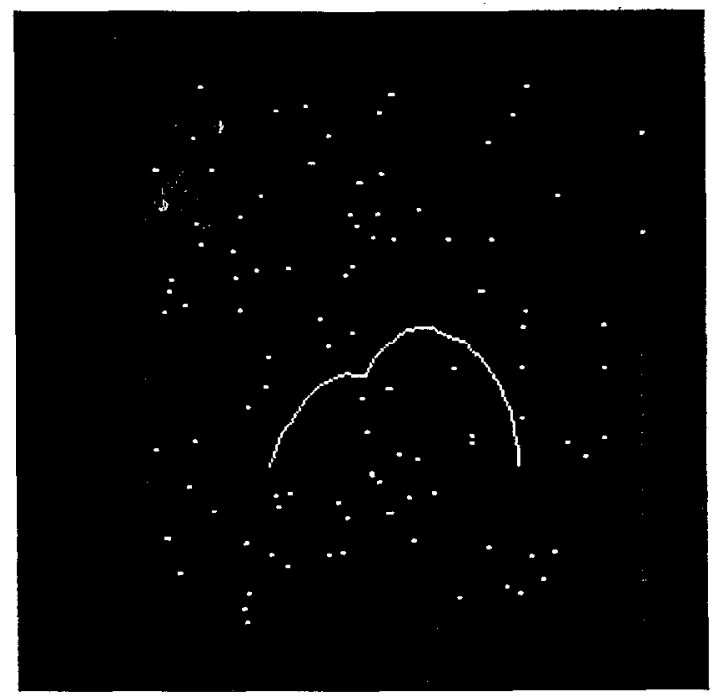

$20 \mathrm{MPa}$

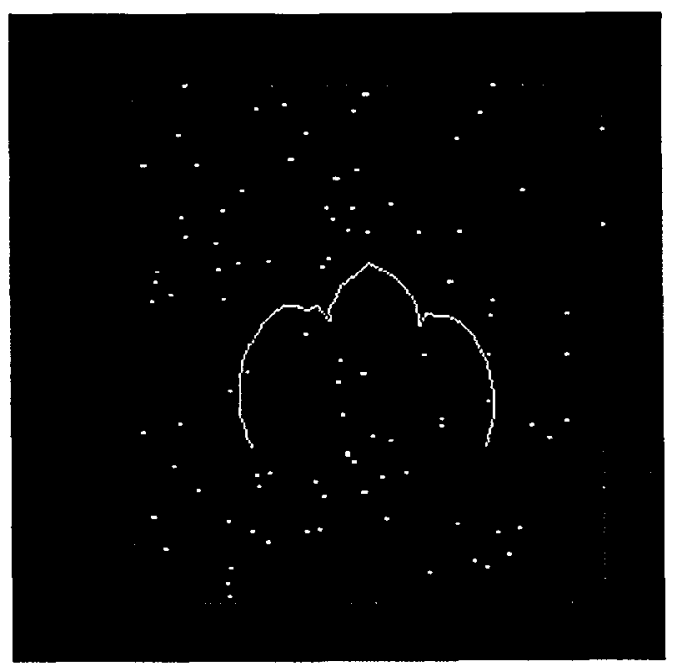

$30 \mathrm{MPa}$

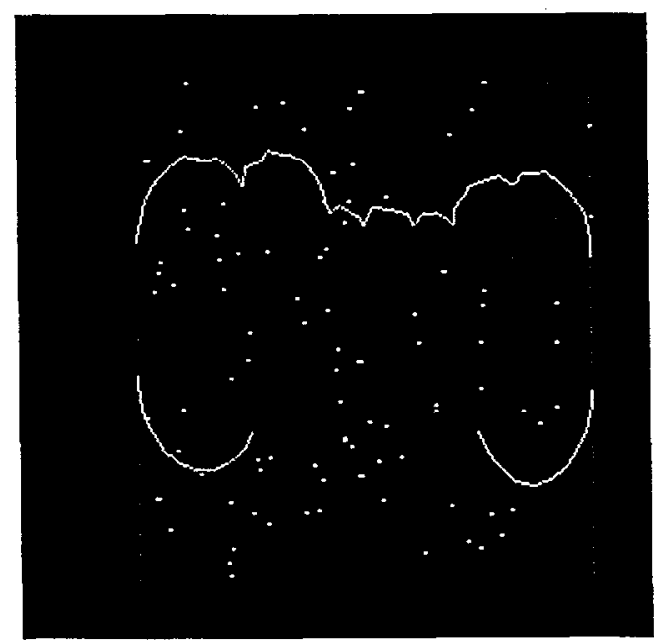

$45 \mathrm{MPa}$

Figure 12. Dislocation percolation around loops. The stand-off distance between the plane of the dislocation and the plane of the loops is $5 \mathrm{~b}$. 


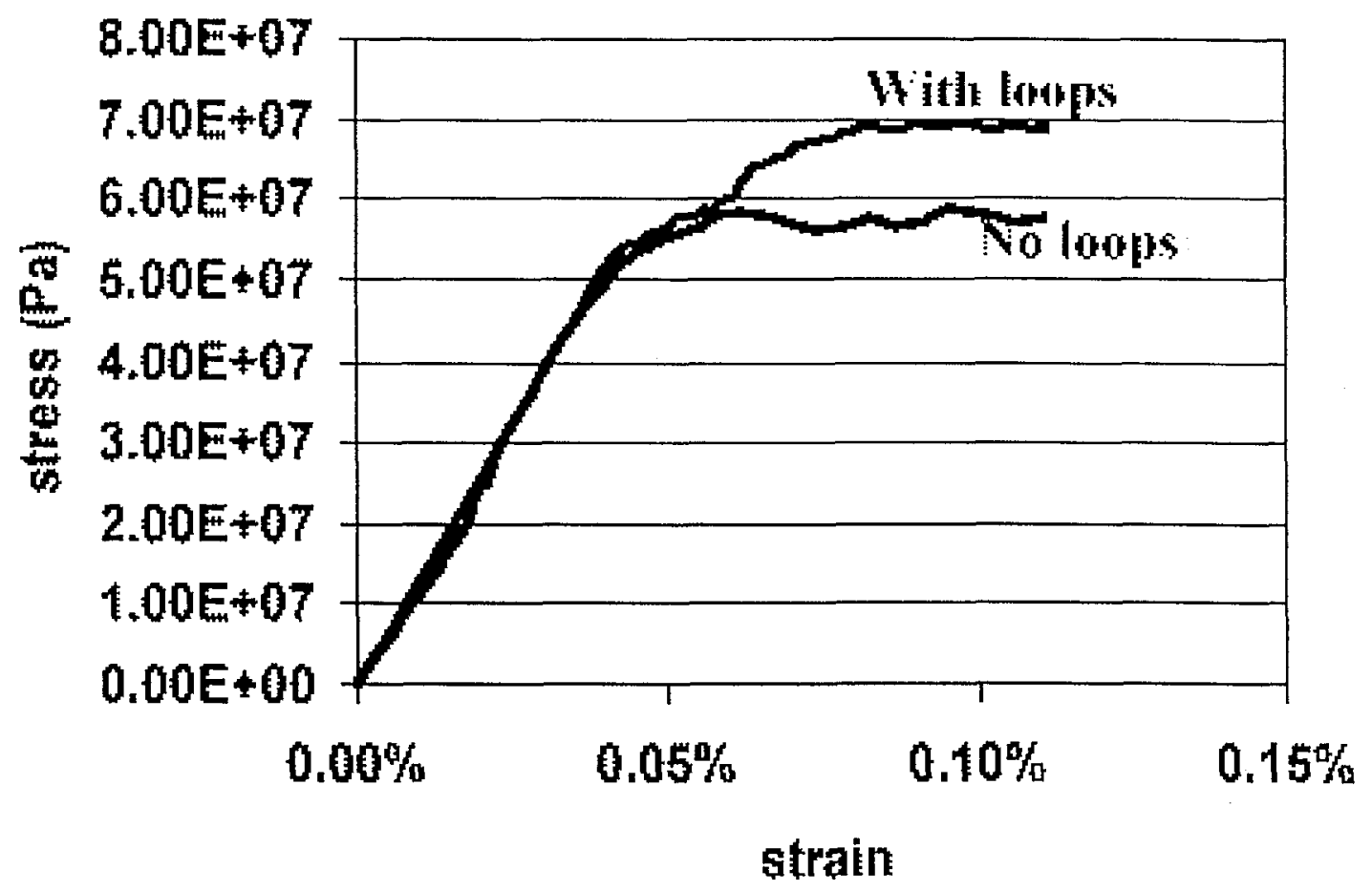

Figure 13. Predicted stress-strain curve showing the effect of dislocation loops in irradiated materials $(\mathrm{Cu})$ on yield stress. 